\title{
Optical Coherence Tomography Angiography Features of Macular Epiretinal Neovascularization In Eales' Disease: A Case Rreport
}

Nora Alyousif ( $\square$ nora_alyousif@hotmail.com )

King Khaled Eye Specialist Hospital https://orcid.org/0000-0001-5399-887X

Abrar K. Alsalamah

KKESH: King Khaled Eye Specialist Hospital

Hassan Aldhibi

King Khaled Eye Specialist Hospital

\section{Brief report}

Keywords: Eales disease, epiretinal neovascularization, optical coherence tomography angiography

Posted Date: August 31st, 2021

DOl: https://doi.org/10.21203/rs.3.rs-824282/v1

License: (1) This work is licensed under a Creative Commons Attribution 4.0 International License.

Read Full License 


\section{Abstract}

Background: Eales disease primarily affects the peripheral retina. However, posterior involvement can be seen. Macular epiretinal neovascularization is not commonly seen in Eales disease. This report highlights the morphology and origin of macular epiretinal neovascularization (ERN) using multimodal retinal imaging, including optical coherence tomography angiography (OCTA).

Results: A 35-year-old man with no history of systemic disorders presented with gradual decrease of vision in his left eye. Fundus examination of his right eye showed peripheral sclerosed blood vessels, neovascularization of the optic disc and elsewhere, and macular ERN. The view of the left fundus was limited by vitreous haemorrhage. Fluorescein angiography (FA), of the right eye showed widespread peripheral capillary nonperfusion and leakage of dye from the retinal neovascularization and macular ERN. Macular Spectral domain optical coherence tomography (SD-OCT) of the right eye showed an epiretinal membrane and the presence of epiretinal neovascular lesions extending above the internal limiting membrane towards the vitreous. Optical coherence tomography angiography (OCTA) showed multiple tiny blood vessels at the macula that arose from the superficial retinal capillary plexuses and extended toward the vitreous. The corresponding B-scan showed flow signal through these vessels and the signal extend above the internal limiting membrane. Systemic work-up was negative except for strongly positive tuberculin skin testing giving the classic diagnosis of Eales disease. Patient was started on empirical anti-tubercular therapy and oral corticosteroids. Scatter laser photocoagulation was applied to nonperfused retinal zones. Despite adequate scatter laser ablation, the ERN failed to regress fully.

Conclusions: Macular ERN can be seen in cases of classic Eales disease. The origin of macular ERN in our case was shown to be from the superficial retinal capillary plexuses. We also noted the slower regression rate of macular ERN as compared to the major neovascularizations of the optic disc and peripheral retina. Further research is needed to establish the pathogenesis of ERN and its optimal management.

\section{Introduction}

Eales disease is characterized by retinal vasculitis, vascular occlusions, subsequent peripheral retinal neovascularization, with or without optic nerve head neovascularization, and recurrent episodes of vitreous haemorrhage [1]. The condition is strongly associated with Mycobacterium tuberculosis, but it remains a diagnosis of exclusion, since it can be indistinguishable from other conditions with retinal neovascularization or inflammation [2].

Although Eales disease primarily affects the peripheral retina, posterior involvement can be seen. Macular edema is the most common macular abnormality, followed by epiretinal membranes, and macular thinning and holes formation [3]. Retinal exudates and haemorrhages has been observed in some cases [3], [4]. 
Herein we describe a classic case of Eales disease that featured an uncommon finding of macular epiretinal neovascularization (ERN) and highlight the clinical and multimodal retinal imaging, including optical coherence tomography angiography (OCTA) characteristics of macular ERN which were not discussed previously in the literature.

\section{Case Report}

A 35-year-old immunocompetent man presented with gradual decease of vision in his left eye for 4 days. He had no history of systemic disorders. On ophthalmic examination, the best-corrected Snellen visual acuity was 20/20 in the right eye and 20/300 in the left eye. Intraocular pressure was normal in both eyes. Anterior segment examination of both eyes was unremarkable. Fundus examination of the asymptomatic right eye showed peripheral peri-phlebitis, peripheral retinal neovascularization, fibrous proliferation towards the superior and inferior vascular arcades, neovascularization of the optic disc and macular ERN. (Figure 1). The view of the left fundus was limited by vitreous haemorrhage. B scan ultrasonography of the left eye showed no traction or retinal detachment. Fluorescein angiography (FA) (Topcon TRC-50DX, Topcon Medical Systems, Inc., NJ, US,; Optos PLC, Dunfermline, UK) of the right eye showed widespread peripheral capillary nonperfusion with peripheral arteriovenous shunting and dye leakage from the neovascularization of the optic disc, elsewhere, and macular epiretinal neovascularization. FA of the left eye was limited by fluorescein blockage effect of the vitreous haemorrhage. Macular spectral domain optical coherence tomography (SD-OCT) (Spectralis OCT, Heidelberg Engineering, Inc., Heidelberg, Germany) of the right eye showed an epiretinal membrane and the presence of epiretinal neovascular lesions extending above the internal limiting membrane towards the vitreous. (Figure 2). The view of left eye OCT was obscured by the vitreous haemorrhage. Optical coherence tomography angiography (OCTA) of the right eye showed multiple tiny blood vessels on the macula that arose from the superficial retinal capillary plexuses and extended toward the vitreous. The corresponding B-scan showed flow signal through these vessels and the signal extend above the internal limiting membrane. (Figure 3). Laboratory investigations including complete blood cell count, fasting blood sugar, sickle cell screen, thrombophilia screen, antinuclear antibody, serum angiotensin-converting enzyme, syphilis serology, and QuantiFERON- TB Gold were within normal limits. Chest radiograph was unremarkable. Tuberculin skin test was positive $(15 \times 15 \mathrm{~mm}$ induration). With this constellation of findings, the diagnosis of Eales disease was made.

Patient was started on oral Prednisolone at a dose of $1 \mathrm{mg} / \mathrm{kg}$ body weight, and was tapered by $5 \mathrm{mg}$ per week for 8 weeks and was given empirical anti-tubercular therapy. Adequate laser photocoagulation guided by FA was applied to areas of nonperfused retina of both eyes with a spot size of 200 micrometres, power of $370 \mathrm{MW}$ and time duration of 0.2 seconds. Laser treatment was started in the left eye, but was limited by the vitreous haemorrhage. During the follow up sessions, vitreous haemorrhage has totally resolved and more laser was applied. The vision has improved to 20/25 in the left eye. After 6 months, the neovascularization has regressed in both eyes. We noted that macular ERN of the right eye required more extensive laser to show evidence of regression (Figure 4). 


\section{Discussion}

In this report, we describe a patient with Eales disease associated with macular epiretinal neovascularization and highlight the clinical and multimodal retinal imaging features. Our patient presented with right fibrovascular proliferation and left dense vitreous haemorrhage, which is classified according to the Eales' disease classification system into Stage 3a and 3b respectively[5]. The growth of neovascularization in the right eye was not only limited to the periphery and optic disc, but also involving the superior and nasal parts of the macula. The macula revealed multiple neovessels, seen on OCT as homogenous multiple lesions penetrating the inner limiting membrane (ILM) and protruding towards the vitreous causing retinal shadowing. These neovascular complexes leaks on FA. They arise from the superficial retinal capillary plexuses and extend into the inner retinal layers, breaching the ILM as seen by OCTA. Flow signal was noted in these tiny macular neovessels which extends above the ILM. This finding on OCTA was not shown before in the literature.

K. Rajurkar and his colleagues reviewed the OCTA features of central Eales disease in case series, they noticed a common finding of deep capillary plexus changes but they did not have a patient in their series with macular ERN in which the origin and morphology of these tiny macular neovessels were shown [6].

ERN was described in cases of diabetic retinopathy, retinal venous occlusion, and recently in cases of macular telangiectasia type 2 and retinitis pigmentosa [7], [8], [9]. In diabetic retinopathy, the neovascularizations was classified by OCTA based on morphology and relationships of retinal neovascular complexes and the posterior vitreous into three types: flat, forward configuration into the vitreous, similar to the configuration in our patient, and tabletop [10]. However, our patient had no history of systemic disorders, including diabetes and hypertension.

OCTA images provide a valuable information about the site of origin of neovascularizations. In diabetes, the neovascularizations were shown to be originated from 3 sites, the most common is from retinal vein adjacent to non-perfused retinal zones; some of the neovascularizations originated from the capillary within the non-perfused retinal area and breached the ILM at multiple sites; other developed from preexisting IRMAs [11]. In our patient the epiretinal neovascularizations originate from superficial retinal capillaries plexus as shown by OCTA, similar to the origin of epiretinal neovascularisations in case of inferotemporal branch vein occlusion (BVO). However, it differs from our case in that the FA in BVOassociated ERN has given smokestack pattern of leakage which might indicate the association with highflow collaterals[8].

Recently, ERN was reported in cases of macular telangiectasia type 2 . it was noticed that these neovascular membranes were communicated with intraretinal plexus associated with collapse of all inner retinal layers resulting in severe diffuse retinal thinning. The hypothesis behind this was that extreme retinal thinning led to the baring of the abnormal capillaries on the retinal surface. However, in our case there was no retinal thinning or any collapse of retinal layers. Furthermore, there are two old reported cases of ERN in young men without significant evidence of systemic diseases [10]. The epiretinal fronds of neovascularization were localized superonasal extending from the optic disc to the equator and 
associated with sectoral retinal ischemia. In one of the cases, there was white sheathing along the vessels which might be similar to vascular sheathing seen in retinal vasculitis. In both cases, the systemic work-up was negative, but they did not look for tuberculin skin test or QuantiFERON level [12]. These two old cases might be similar to our case, but the location of ERN differs. In addition, the ERN was not confirmed by OCT and OCTA as the article is old. OCTA in our case, clearly showed the origin and morphology of these ERN.

Posterior ERN was also seen in cases of retinitis pigmentosa. Intravitreal anti-VEGF treatment was used which resulted in regression of neovascularization on fundus examination but persisted on OCTA [8]. It is worth studying the effect of adding anti-VEGF to scatter laser photocoagulation in the treatment of ERN as hypoxia might play a role in the mechanism of formation. In our case, we did not consider giving the patient intravitreal anti-VEGF agents as his vision was 20/20 and it is an invasive procedure that might be associated with significant adverse events.

Our patient received multiple sessions of laser photocoagulation to the nonperfused retinal zones. This has induced regression of neovascularization of the optic disc and at the periphery. However, the regression rate was slower in epiretinal macular neovascularization and the flow signal through the tiny blood vessels remained evident on OCTA. This finding might suggest that the activity of epiretinal neovascularization can be masked on fundus examination and FA, making OCTA a better tool for monitoring the activity of ERN. Little is known about the mechanism of formation of ERN. We believe that ischemia might play a role as ERN showed evidence of regression with the addition of more laser targeting the nonperfused retinal areas. Further research is needed to establish the pathogenesis of ERN and its optimal management.

\section{Abbreviations}

Epiretinal neovascularization (ERN)

Fluorescein angiography (FA)

Spectral domain optical coherence tomography (SD-OCT)

Optical coherence tomography angiography (OCTA)

Branch vein occlusion (BVO)

\section{Declarations}

\section{Ethics declarations:}

Ethics approval and consent to participate: This report was approved by the Institutional Review Board of King Khaled Eye Specialist Hospital (KKESH) and adhered to the tenets of the Declaration of Helsinki. 
Consent for publication: Consent to publish the case report has been taken from the patient concerned and does not disclose the identity or infringe the privacy of the patient.

Availability of data and material: The datasets used during the current study are available from the corresponding author on reasonable request.

Competing interests: The authors declare that they have no competing interests

Funding: Not applicable.

Authors' contributions: HA provided ophthalmic care to the patient and drafted the manuscript. NA and AA drafted the manuscript and reviewed the literature. All authors have reviewed the manuscript and approved the same.

Acknowledgements: Not applicable

\section{References}

[1] T. Das, A. Pathengay, N. Hussain, and J. Biswas, “Eales' disease: Diagnosis and management," in Eye, Jan. 2010, vol. 24, no. 3, pp. 472-482, doi: 10.1038/eye.2009.315.

[2] P. Gupta and J. Biswas, "Further evidence of the association of latent Mycobacterium tuberculosis in Eales' disease," Int. Ophthalmol., pp. 1-6, Nov. 2020, doi: 10.1007/s10792-020-01645-5.

[3] N. Goel, V. Kumar, S. Arora, P. Jain, and B. Ghosh, "Spectral domain optical coherence tomography evaluation of macular changes in Eales disease," Indian J. Ophthalmol., vol. 66, no. 3, pp. 433-438, Mar. 2018, doi: 10.4103/ijo.IJO_845_17.

[4] S. Saxena and D. Kumar, "Macular involvement Eales disease," Ann. Ophthalmol. - Glaucoma, vol. 32, no. 2, pp. 98-100, Jun. 2000, doi: 10.1007/s12009-000-0024-3.

[5] S. Saxena and D. Kumar, "New classification system-based visual outcome in Eales' disease," Indian J. Ophthalmol., vol. 55, no. 4, pp. 267-269, 2007, doi: 10.4103/0301-4738.33038.

[6] K. Rajurkar, M. Thakar, P. Gupta, and A. Rastogi, “Comparison of fundus fluorescein angiography, optical coherence tomography and optical coherence tomography angiography features of macular changes in Eales disease: a case series," J. Ophthalmic Inflamm. Infect., vol. 10, p. 34, 2020, doi: $10.1186 / \mathrm{s} 12348-020-00220-4$.

[7] A. G. Ayachit, L. U. Reddy, S. Joshi, and G. S. Ayachit, “Epiretinal Neovascularization: A Novel OCT Angiography Finding in Macular Telangiectasia Type 2," Ophthalmol. Retin., vol. 3, no. 6, pp. 516-522, Jun. 2019, doi: 10.1016/j.oret.2019.01.022. 
[8] S. Verma, P. Venkatesh, and V. Kumar, "Smokestack Leak from Retinal Neovascularization," Ophthalmology Retina, vol. 5, no. 2. Elsevier Inc., p. 177, Feb. 01, 2021, doi: 10.1016/j.oret.2020.10.014.

[9] J. Lee, J. Han, and S. H. Byeon, "Retinitis Pigmentosa with Epiretinal Neovascularization at the Macula," Ophthalmology Retina, vol. 5, no. 4. Elsevier Inc., p. 329, Apr. 01, 2021, doi: 10.1016/j.oret.2020.12.021.

[10] S. Vaz-Pereira, K. K. Dansingani, K. C. Chen, M. J. Cooney, J. M. Klancnik, and M. Engelbert, "TOMOGRAPHIC RELATIONSHIPS between RETINAL NEOVASCULARIZATION and the POSTERIOR VITREOUS in PROLIFERATIVE DIABETIC RETINOPATHY," Retina, vol. 37, no. 7, pp. 1287-1296, Jul. 2017, doi: 10.1097/IAE.0000000000001336.

[11] J. Pan et al., "Characteristics of Neovascularization in Early Stages of Proliferative Diabetic Retinopathy by Optical Coherence Tomography Angiography," Am. J. Ophthalmol., vol. 192, pp. 146-156, Aug. 2018, doi: 10.1016/j.ajo.2018.05.018.

[12] S. H. Sinclair and T. A. Meredith, "Posterior epiretinal neovascularization associated with sectoral retinal ischemia," Retina, vol. 3, no. 3, pp. 188-193, 1983, doi: 10.1097/00006982-198300330-00008.

\section{Figures}

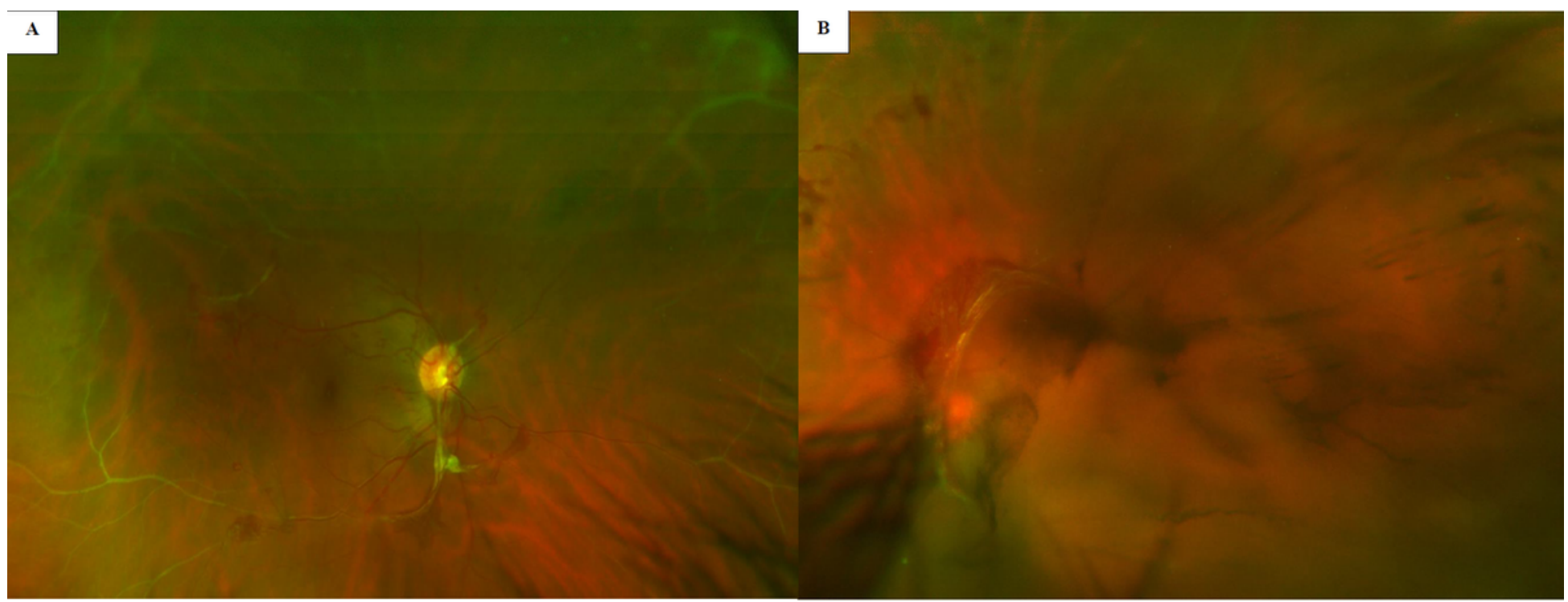

\section{Figure 1}

Fundus photograph at presentation showing right eye $(A)$ perivenous sheathing, fibrous proliferation along the superior and inferior vascular arcades, neovascularization of the optic disc and macular ERN. The view of the left eye (B) is limited by vitreous haemorrhage 


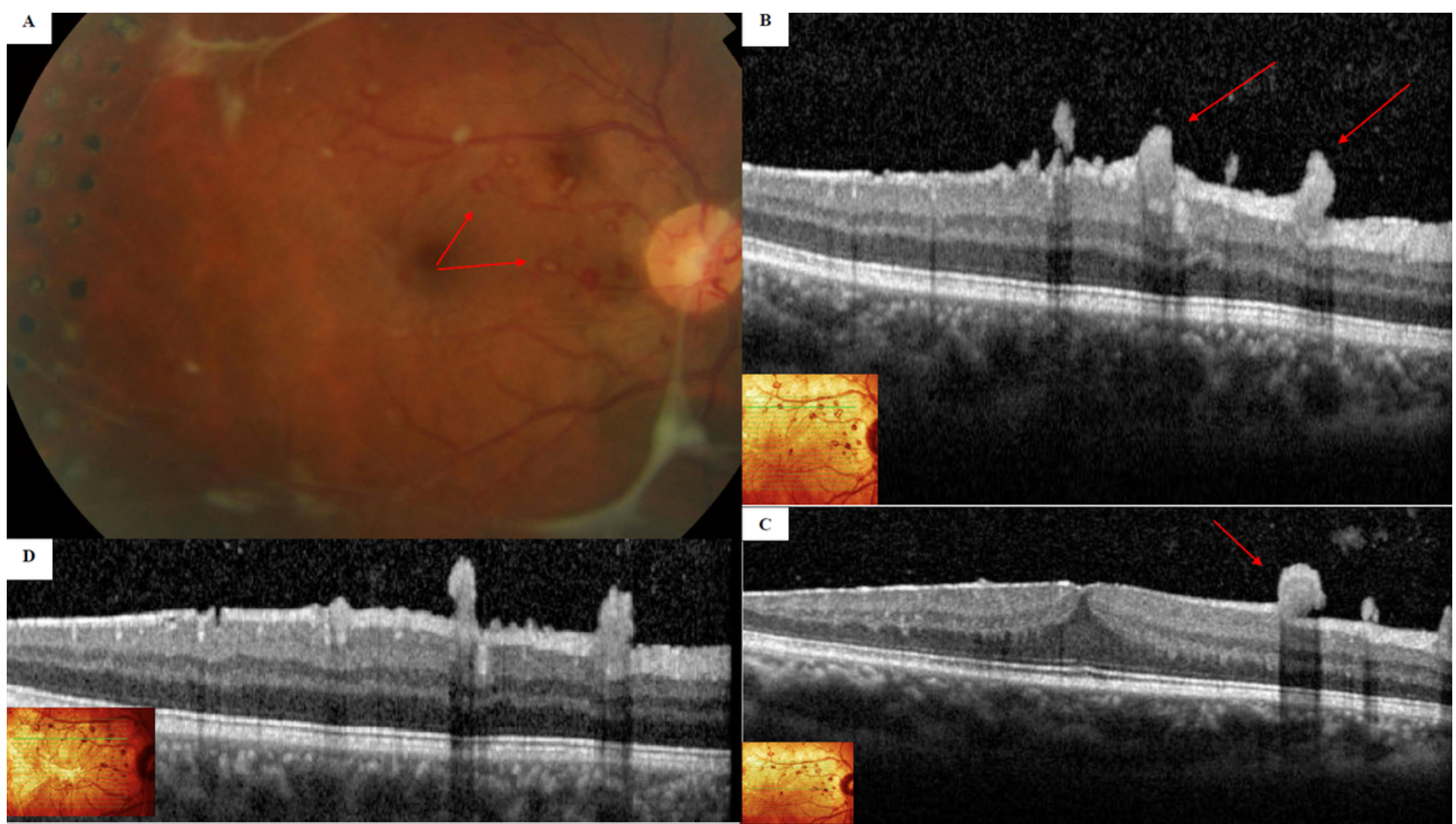

\section{Figure 2}

Colour fundus image (A) of the right eye showing multiple tiny blood vessels at the macula (Red arrows). Optical coherence tomography $(O C T)$ of the right macula at different cuts $(B, C, D)$ showing multiple tiny hyperreflective lesions extending above the ILM toward the vitreous (Red arrows) causing retinal shadowing with intact retinal layers and reflectivity. 


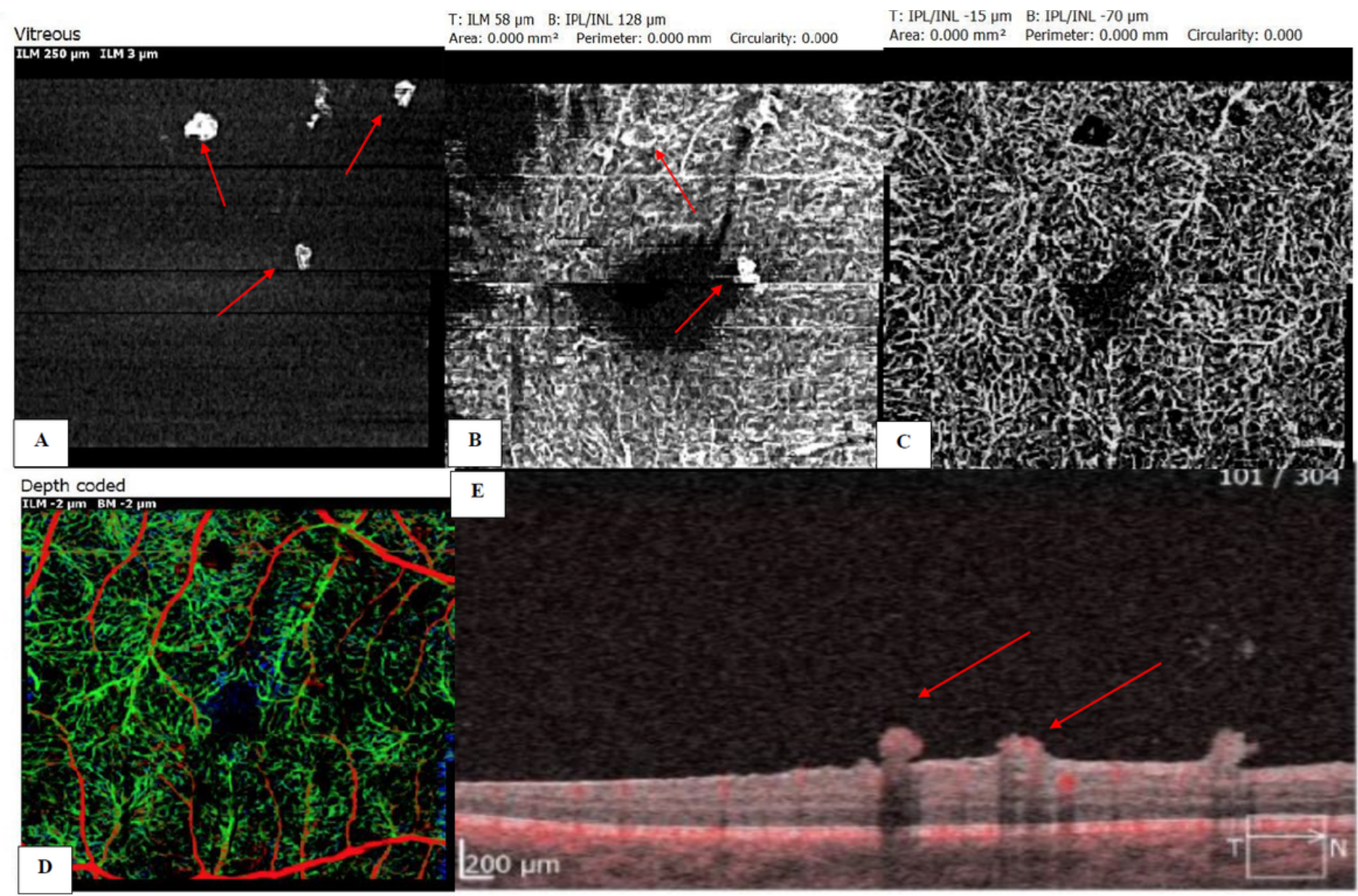

\section{Figure 3}

Optical coherence tomography angiography (OCTA) of the right eye showing multiple tiny blood vessels at the macula that arose from the superficial retinal capillary plexuses as seen by superficial retinal slab and extended towards the vitreous $(A, B)$ (arrows). The tiny blood vessels disappeared in the deep retinal slab (C). The Depth coded view and corresponding B-scan showed flow signal through these tiny macular blood vessels and the signal extends above the ILM (arrows) (D,E). 


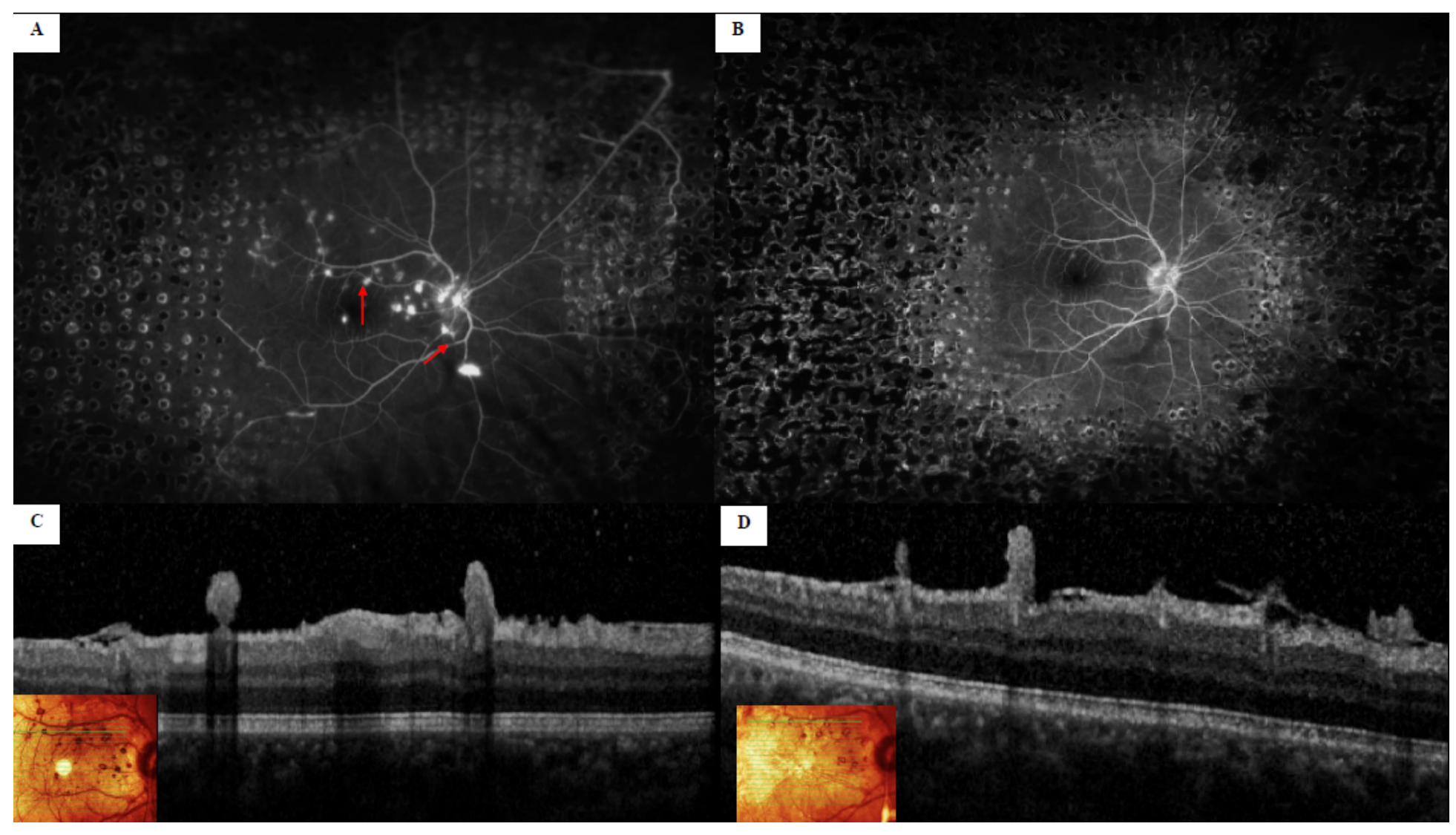

\section{Figure 4}

Ultra-widefield late-frame fluorescein angiography of the right eye (A), showing scatter laser photocoagulation marks to the nonperfused retinal zones with regression of peripheral neovascularization. Note the persistence of ERNM (red arrows). Corresponding OCT (C) showing multiple hyperreflective lesions extending above the ILM towards the vitreous. During the follow up sessions and after the addition of more laser marks, (B) No more leakage of dye from ERN. Corresponding OCT (D) showing shrinkage in lesions size as compared to (C). 